\title{
Efecto del entrenamiento de fuerza en deportistas femeninas de deportes colectivos mediante tecnología isoinercial
}

\author{
Effect of strength training on female team sports \\ athletes using isoinertial technology
}

\author{
Salvador Pérez Muñoz*, David Morilla de la Riva, Gema Alonso García, Antonio Sánchez Muñoz, Francisco José Albert \\ García y Alberto Rodríguez Cayateno
}

Universidad Pontificia de Salamanca. Facultad de Educación. Grupo de investigación EGIIOFID.

\begin{abstract}
Resumen: El objetivo de esta investigación es analizar el efecto de un programa de fuerza mediante tecnología isoinercial durante cuatro semanas, en la mejora del rendimiento en mujeres de deportes colectivos con carácter intermitente. La muestra estaba compuesta por seis mujeres con una edad media de $21 \pm 1.79$ ańos. Se realizaron los test de cambios de dirección $V$-cut, fuerza explosiva counter movement jump y repeated sprint ability sobre $40 \mathrm{~m}$. Los principales resultados muestran que se mejoraron todas las variables analizadas mediante el entrenamiento con tecnología isoinercial. Además, presentaron diferencias significativas $(\mathrm{p}<.05)$ en las variables de fuerza explosiva y de repeated sprint ability. Por lo tanto, un programa de entrenamiento de fuerza a través de tecnología isoinercial durante cuatro semanas de entrenamiento es suficiente para la mejora de los cambios de dirección, la resistencia a la repetición de esprines y la fuerza explosiva de piernas.

Palabras clave: Fuerza explosiva; Entrenamiento Isoinercial; Deportes co-
\end{abstract}

lectivos; Mujeres.

Abstract: The aim of this research is to analyse the effect of a four-week strength programme using isoinertial technology on performance improvement in women in team sports on an intermittent basis. The sample was composed of six women with an average age of 21 ( \pm 1.79 years). The tests of V-cut direction changes, explosive force counter movement jump and repeated sprint ability over $40 \mathrm{~m}$ were performed. The main results show that all the variables analysed are improved by training with isoinertial technology. They present significant differences $(p<.05)$ in the variables of explosive strength and repeated sprint ability. Therefore, a strength training program using isoinertial technology during four weeks of training is sufficient for the improvement of direction changes, resistance to repeated sprinting and explosive leg strength.

Keywords: Explosive force; Isoinertial training; Collective sports; Women.

\section{Introducción}

Los deportes colectivos son los que cuentan con más licencias deportiva en España, destacando fútbol y baloncesto (CSD, 2018). El primero es, por supuesto, el deporte más practicado, destacando sobre los demás en el caso del fútbol masculino (Pérez, Sánchez \& Urchaga, 2015), no siendo así en jugadoras. Ahora bien, en los últimos años el deporte femenino en general está experimentado un crecimiento importante, no sólo en los deportes colectivos, sino también en otros deportes.

Los deportes de equipo son aquellos en lo que se produce una interacción con jugadores del equipo propio y con jugadores del equipo rival. En este sentido, se requiere inevitablemente, de un gran bagaje de factores que intervienen en el juego y en el rendimiento, como son los factores psicológicos, técnicos, tácticos y físicos (Garganta, 2001; Jones \& Drust, 2007). Por tanto, el entrenamiento ha de ser adaptado y específico al propio deporte.

En este sentido, en todos los deportes de equipo, como por ejemplo baloncesto, fútbol, balonmano, entre otros. La exigencia es muy alta a nivel funcional y fisiológico (Núñez,

Dirección para correspondencia [Correspondence address]: Salvador Pérez Muńoz. Universidad Pontificia de Salamanca (España).

E-mail: sperezmu@upsa.es
Poblador \& Ramírez, 2016), se producen fases de aceleración y desaceleración, arrancadas, paradas, cambios de dirección (COD) de carácter intermitente, etc. (Bangsbo, Mohr, \& Krustrup, 2006; Cabanillas, Serna, Muñoz-Arroyave \& Echeverri, 2020; Fiorilli et al., 2020; Irigoyen, Huerta, Alvira, Benito, \& Larumbe, 2014; Pardeiro \& Janci, 2017; Romero, Feria, Sañudo, De Hoyo \& Del Ojo, 2014; Santiago, Granados, Quintela \& Yanci, 2015). Normalmente, todos los deportistas tanto de deportes de equipo como individuales utilizan en sus entrenamientos de fuerza cargas concéntricas y excéntricas en el plano vertical (Santiago et al., 2015), y rara vez utilizan entrenamientos con carga excéntrica en el plano horizontal u otros planos (Monajati, Larumbe-Zabala, Sampson, \& Naclerio, 2018; Tous-Fajardo, Gonzalo-Skok, Arjol-Serrano, \& Tesch, 2016).

Con el objetivo de mejorar los COD, algunos de los programas de entrenamiento incluyen ejercicios de velocidad lineal, parecen dar buenos resultados en la mejora de las habilidades de sprint y salto en atletas jóvenes, pero no en los más experimentados. Sin embargo, tal habilidad parece dictar el rendimiento y discriminar los jugadores competitivos (TousFajardo et al., 2016).

Las exigencias asociadas con la aceleración, desaceleración 
y rápidos COD son considerados en la literatura científica como un componente clave en las demandas del juego, como por ejemplo en estudios de fútbol (Bangsbo et al., 2006; Pardeiro \& Yanci, 2017; Santiago et al., 2015; Pérez et al., 2019) y en otros deportes y poblaciones (Cabanillas et al., 2020; Suarez-Arrones et al., 2020; Yanci, Castillo, Vizcay, Pitillas \& Iturricastillo, 2016). A esta capacidad motora compleja, conocida en la literatura anglosajona como agilidad (SuarezArrones et al., 2020), se le otorga una gran importancia en los deportes de equipo, al permitir a los jugadores realizar acciones especificas como driblar a un defensor en una acción de ataque o presionar en tareas defensivas (Gonzalo-Skok, 2015), si bien, actualmente es conocido más por el término de COD.

Uno de los grandes factores determinantes en los deportes de equipo son los COD, en los cuales reside el éxito de muchos jugadores y que se relacionan con la fuerza excéntrica de la musculatura flexora de la rodilla o con la fuerza excéntrica de todo el tren inferior (Tous-Fajardo et al., 2016). En este sentido, Gonzalo-Skok (2015) expone que en jugadores de baloncesto realizaban $105^{\circ}$ movimientos por partido, además, explica los cambios suponen un cambio de acción cada dos segundos, siendo los movimientos más específicos como las carreras en diagonal, los reversos y giros las acciones que más veces se repetían, lo que confirmaría la importancia de las acciones donde se realizaba algún COD.

Ahora bien, para poder mejorar o desarrollar los COD los entrenamientos se deberían adaptar, ya que los entrenamientos tradicionales o convencionales, en los que los sujetos utilizan sobrepesos pueden provocar lesiones debido a las sobrecargas excéntricas (Núñez et al., 2016). Sin embargo, existe otro método de entrenamiento que permitiría mejorar notablemente la fuerza excéntrica en los deportistas, eso sí utilizan la tecnología isoinercial la cual, va a permitir adaptaciones musculares fuertes y tempranas, en mayor medida que el entrenamiento con pesas tradicionales (Cabanillas et al., 2020; Fiorilli et al., 2020; Prieto-Mondragón, Camargo-Rojas, \& Quiceno, 2016) o entrenamientos pliométricos (Fiorilli et al., 2020). La magnitud de estos efectos provoca que la mejora de la fuerza, así como de la potencia y el tamaño muscular, sea mayor utilizando tecnología isoinercial que utilizando pesos de gravedad dependiente (Cabanillas et al., 2020; Fiorilli et al., 2020; Núńez et al., 2016; Romero et al., 2014).

Los entrenamientos realizados mediante dispositivos inerciales utilizan la resistencia de inercia rotacional ofreciendo resistencia independiente de la gravedad, utilizando volantes giratorios durante el movimiento para generar la fuerza de inercia, con mayor carga excéntrica, que permite realizar movimientos en las tres dimensiones del espacio, adaptados individualmente y difiere de entrenamientos tradicionales, que utilizan principalmente el peso libre (Cabanillas et al., 2020; Fiorilli et al., 2020; Monajati et al., 2018; Suarez-Arrones et al., 2018; Prieto-Mondragón et al., 2016; Vázquez-Guerrero
\& Moras, 2015). Estos dispositivos están formados por un volante de rotación inercial, además de un eje que está fijo sobre el que giran las masas o pesos que pueden variar hasta un total de 16 pesos y, además, modifica la velocidad (Núñez et al., 2019). En un primer movimiento ocurre la fase concéntrica cuando la cuerda se desenrolla totalmente y cuando se recoge se trabaja la fase excéntrica (Vázquez-Guerrero \& Moras, 2015), en este caso no hay fuerza de fricción lo que permite que ambas fuerzas sean iguales, permitiendo un gran esfuerza excéntrico y sin embargo con menor costo metabólico (Fiorilli et al., 2020).

De esta forma según Cabanillas, et al. (2020), Douglas, Pearson, Ross y McGuigan (2017), Fiorilli et al. (2020), Monajati, et al. (2018) y Petré, Wernstål y Mattson (2018) el entrenamiento con volante inercial es una modalidad actual de entrenamiento de fuerza que mejora el rendimiento de los deportistas y ofrece la posibilidad de realizar ejercicios con sobrecarga excéntrica y resistencia variable, en comparación con los métodos tradicionales basados en la resistencia de la gravedad. Además, se menciona que el entrenamiento con volante inercial parece una alternativa viable para la mejora de la fuerza (Cabanillas et al., 2020; Monajati et al., 2018; Norrbrand, Pozzo \& Tesch, 2010; Petré et al., 2018; PrietoMondragón et al., 2016), mejora frente a los entrenamientos tradicionales (Cabanillas et al., 2020; Douglas et al., 2017) y produce adaptaciones hipertróficas en sujetos no entrenados, moderadamente entrenados y sujetos entrenados, así como mejoras en la coordinación intramuscular, menos estrés cardiovascular, mayor potencia y rendimiento muscular en comparación con las cargas libres, y presenta un efecto protector que dura varios meses (Cabanillas et al., 2020; Douglas et al., 2017).

En esta línea hay varios estudios que demuestran que el uso de la tecnología inercial es eficiente, mejorando el rendimiento y entrenamiento deportivo, ayudan a la prevención y rehabilitación de lesiones deportivas (Cabanillas et al., 2020; Monajati et al., 2018; Norrbrand et al., 2010; Núñez et al., 2016; Prieto-Mondragón et al., 2016; Tous-Fajardo et. al., 2016), además de mejorar en mayor medida que aquellos que hacen entrenamientos de pesas convencionales (Cabanillas et al., 2020; Monajati et al., 2018) o pliométricos (Fiorilli et al., 2020).

Por último, los estudios centrados en mujeres de forma exclusiva son escasos ya sea en fútbol o en otros deportes, por ello consideramos adecuado investigar en mujeres que juegan en deportes colectivos. Por lo tanto, el objetivo del estudio fue analizar el efecto de un programa de fuerza mediante tecnología isoinercial durante cuatro semanas en la mejora del rendimiento en mujeres de deportes colectivos con carácter intermitente. 


\section{Metodología}

La investigación realizada es de tipo cuantitativa, donde se realiza un pretest, la intervención y un postest final, para comprobar el efecto de la intervención sobre la fuerza y los cambios de dirección. Las características de este tipo de metodología es que los datos sean observables, medibles, cuantificables y se ofrecen unos resultados numéricos (Cook \& Reichardt, 1986; Pita \& Pértegas, 2002).

\subsection{Participantes}

La muestra está compuesta por seis jugadoras de fútbol sala y baloncesto, con una edad media de $21 \pm 1.79$ años), $57.5 \pm 7.26$ $\mathrm{kg}$ de peso, altura de $164.6 \pm 7.31$ centímetros, índice de masa corporal (IMC) de 21.28 \pm 3.37 , tienen $14.17 \pm 4.35$ ) ańos de experiencia en el deporte y realizan una media de $4.33 \pm 1.50$ ) horas de entrenamiento a la semana. Alumnas todas ellas del Grado en Ciencias de la Actividad Física y del Deporte en tercer curso, representando el $54.5 \%$ de la población femenina total, si bien no se realiza comparación entre el grupo que forma parte del estudio y el que no lo forma. En este caso, por motivos operativos y de facilidad de acceso a la muestra, las participantes fueron seleccionados mediante muestreo no aleatorio incidental. En todos los casos participaron de forma voluntaria, autorizada y no recompensada.

\subsection{Procedimiento}

Los datos fueron recogidos en las instalaciones de la Universidad Pontificia de Salamanca con previa solicitud y autorización a los responsables de la Universidad y de las jugadoras participantes en el estudio, todas ellas mayores de edad, aceptando la participación de forma voluntaria.

El estudio fue aprobado por el Comité Ético de la Universidad Pontificia de Salamanca y se llevó a cabo siguiendo escrupulosamente las normas deontológicas reconocidas en la Declaración de Helsinki 2013. Convenio de Oviedo y siguiendo las recomendaciones de Buena Práctica Clínica de la CEE (documento 111/3976/88 de julio de 1990) y la normativa legal vigente española que regula la investigación clínica en humano (Real Decreto 561/1993 sobre ensayos clínicos). Además, se requirió la firma de un documento de consentimiento informado para participar.

En este estudio sólo existe un grupo de trabajo no realizando comparación de resultados con otro grupo. Las deportistas fueron asignadas a cuatro semanas de entrenamiento controlado y planificado, en las que realizaban un entrenamiento semanal con sobrecarga excéntrica a través de tecnología isoinercial.

Todas las deportistas realizaban un calentamiento estandarizado y el mismo protocolo de entrenamiento de fuerza.
Todos los test se realizaron en un pabellón con suelo antideslizante. Antes de comenzar el estudio se les pasó los test y una vez terminado el estudio se realizaron de nuevo los test finales. Se pidió a las deportistas que no realizaran ninguna actividad física intensa en las $48 \mathrm{~h}$ previas a la realización de las pruebas de evaluación.

El entrenamiento se llevó un día por semana, después de realizar un protocolo de calentamiento estandarizado que consistía en: activación aeróbica (siete minutos de carrera continua), movilidad articular dinámica durante siete minutos, cinco minutos de estiramientos estáticos de las extremidades inferiores (uno de cuádriceps, dos de isquiotibiales, uno de aductores, uno de psoas ilíaco, y uno de gemelo) y cuatro aceleraciones cortas (Prieto \& García, 2013). El entrenamiento consistió en una batería de siete ejercicios: A) rotación de tronco B) paso atrás con flexo - extensión de rodilla C) sentadilla unilateral D) sentadilla a una pierna en plataforma inestable E) nordic - hamstring F) planchas frontales y laterales G) Abducción - aducción resistida con compañero (Tous-Fajardo et al., 2016).

La organización de los entrenamientos tuvo la siguiente secuenciación:

- Semana 1: sesión de familiarización con el protocolo de entrenamiento.

- Semana 2: dos series de cuatro repeticiones para los ejercicios realizados con Versapulley ${ }^{\mathrm{TM}}$, dos series de seis repeticiones con cada pierna para sentadilla a una pierna en plataforma inestable, dos series de cinco repeticiones para nordic - hamstring, y 15 segundos para los ejercicios de planchas y abducciones - aducciones resistidas con compañero.

- Semana 3: dos series de seis repeticiones para los ejercicios realizados con Versapulley ${ }^{\mathrm{TM}}$, dos series de ocho repeticiones con cada pierna para sentadilla a una pierna en plataforma inestable, dos series de seis repeticiones para nordic - hamstring, y 20 segundos para los ejercicios de planchas y abducciones - aducciones resistidas con compañero.

- Semana 4: dos series de seis repeticiones para los ejercicios realizados con Versapulley ${ }^{\mathrm{TM}}$, dos series de 10 repeticiones con cada pierna para sentadilla a una pierna en plataforma inestable, dos series de seis para nordic - hamstring, y 25 segundos para los ejercicios de planchas y abducciones - aducciones resistidas con compañero.

En los ejercicios con Versapulley ${ }^{\mathrm{TM}}$ se pidió a las deportistas realizar la fase concéntrica tan rápido como fuera posible y reteniendo la fase excéntrica. La recuperación era pasiva y comprendía un minuto entre serie y dos minutos entre ejercicios. 


\subsection{Instrumentos}

La parte experimental se ha desarrollado en las instalaciones de la Universidad Pontificia de Salamanca, los test fueron medidos por el mismo evaluador tanto al inicio como al final de la investigación, además fue formado este evaluador en la toma de datos para evitar errores. Los participantes pudieron practicar una semana antes la realización de los test en el laboratorio un total de dos intentos en cada uno, con un doble objetivo, familiarizarse con los test para evitar errores en la toma de datos y a su vez preparar al evaluador, similar a otro estudio (Núñez et al., 2019).

Todas las participantes se sometieron a los siguientes tests iniciales y finales, que consistían en:

- $\quad$ Test CMJ (counter movement jump): para este test los sujetos se colocaron en posición erguida, con las manos en las caderas, y tuvieron que realizar un salto con una previa flexo - extensión de las rodillas. Para ello, se tomaron dos medidas:

o Longitud de miembros inferiores: mide la distancia desde el trocánter mayor del fémur hasta la punta del pie, con el sujeto tumbado boca arriba y el tobillo completamente extendido.

o Altura 90\%: mide la distancia vertical entre el trocánter mayor del fémur y el suelo en una posición óptima de flexión de rodilla que permita saltar lo más alto posible (rodilla flexionada a 90). Ambas mediciones se hicieron de la misma extremidad.

o Se realizaron dos saltos, analizando el mejor de ellos mediante la aplicación móvil "My jump 2", aplicación científicamente validada (Balsalobre-Fernández, Glaister, \& Lockey, 2015).

- Test V-Cut: se pidió a los sujetos que realizaran un sprint de 25 metros, realizando cuatro cambios de dirección de $45^{\circ}$. Para que el test fuera válido, los sujetos tenían que pisar entre los conos al realizar el cambio de dirección. Se realizaron dos series, con descanso de tres minutos entre cada serie. En caso de que una se considerara errónea o fallida, se realizó una tercera. Se eligió el mejor tiempo para su análisis (Gonzalo-Skok et al., 2015) (Figura 1).

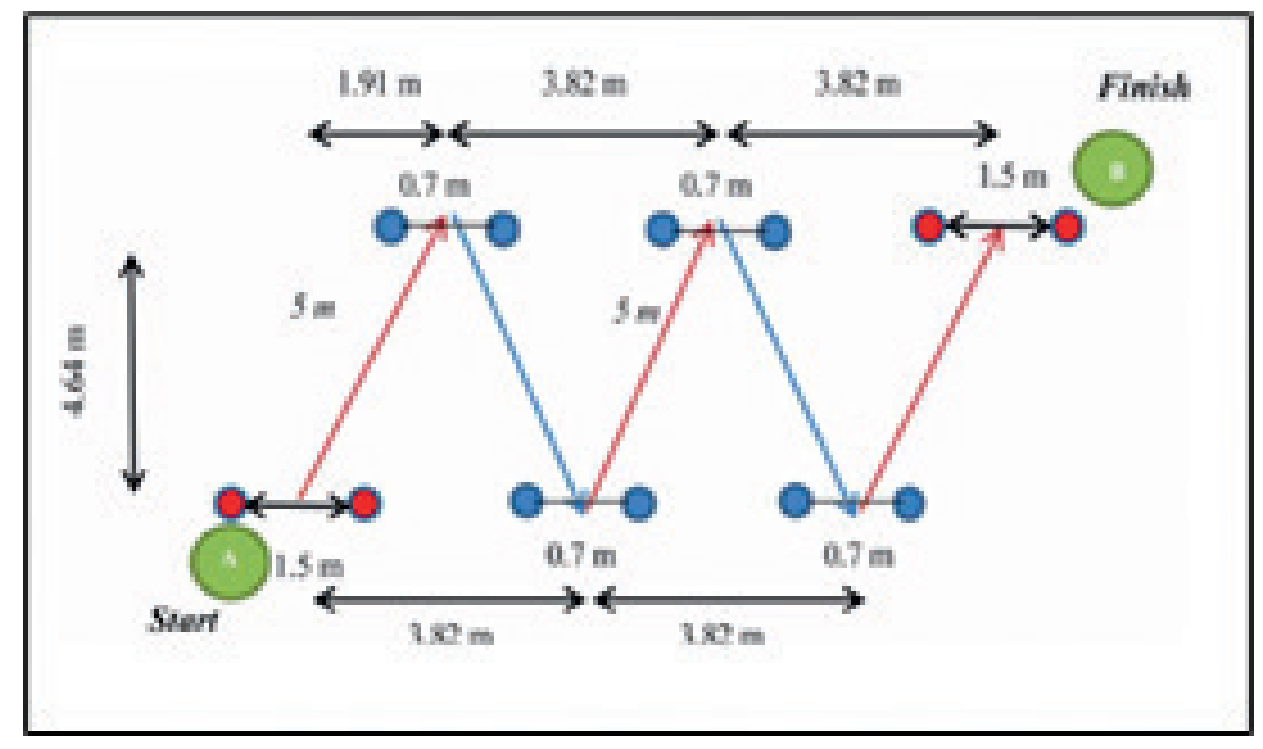

Figura 1. Test V-Cut

- Test RSA (repeated sprint ability): se pidió a los sujetos que realizaran seis esprines de 40 metros, 20 metros de ida y 20 metros de vuelta, con un cambio de dirección de $180^{\circ}$, tocando la línea entre los conos con un pie, y una recuperación pasiva de 20 segundos entre cada sprint realizado. Se eligió la mejor serie, la media de las seis series, y el \% de reducción de los tiempos para análisis (Bishop, Spencer, Duffield, \& Lawrence, 2001).

\subsection{Análisis estadístico de los datos}

En primer lugar, se realizó un análisis exploratorio de los datos transformando las variables en puntuaciones tipificadas. Por otro lado, se analizaron los supuestos subyacentes a la aplicación de la herramienta estadística (i.e., normalidad Shapiro-Wilks). Todas las variables cumplieron con el supuesto de normalidad. La prueba t de Student para muestras dependientes fue realizada para cada una de las variables dependientes. Las diferencias significativas se establecieron a nivel 
de $p<.05$. Se utilizó el software de análisis estadísticos se utilizó SPSS. Se estimó el tamaño del efecto de las interacciones (TE $[d g 2])$ mediante los cambios medios tipificados, donde:

$$
d_{c 2}=c(g l) * \frac{\left(X_{\text {pre }}\right)-\left(X_{\text {post }}\right)}{S_{\text {pre }}}
$$

Donde: $\left.\mathrm{c}_{\mathrm{glE}, \mathrm{C}}\right)$ es la corrección de los grados de libertad, estimada mediante:

$$
\sigma(d c 2)=c(g l)^{2} \times \frac{2 *(1-r)}{n} \times \frac{n-1}{n-3} \times 1+\frac{n * d c 2^{2}}{2 *(1-r)}-d c 2^{2}
$$

\section{Resultados}

Los principales resultados del estudio muestran que en el caso del pretest los resultados nos muestran que en la prueba de fuerza explosiva CMJ la media de los valores es de 22.9 ( \pm 2.14) $\mathrm{cm}$. Para los cambios de dirección (COD) los valores medios obtenidos en el pretest fueron de 8.12 ( \pm .702) segundos. Y finalmente para la repetición de esprines la media obtenida es de 8.65 ( \pm .360) segundos (tabla 1$)$.

Tabla 1. Pretest realizado media y desviación típica: CMJ, RSA y COD.

\begin{tabular}{lccc}
\hline & N & Media & DT \\
\hline CMJ Pretest & 6 & 22.9 & 2.14 \\
COD Pretest & 6 & 8.12 & .702 \\
RSA Pretest & 6 & 8.65 & .360 \\
\hline
\end{tabular}

CMJ: Counter Movement Jump / RSA: Repeated Sprint Ability / COD: Cambios de Dirección V-Cut.

En cuanto a los descriptivos, tras realizar la intervención como se muestran en la tabla 2 , los resultados muestran que en el caso de la fuerza explosiva-CMJ los valores medios son $27.35( \pm 3.17) \mathrm{cm}$. En los COD la media que se obtuvo fue de 7.67 ( \pm .219$)$. Por último, en la RSA la media es de 8.41 $( \pm .360)$.

Tabla 2. Postest realizado, media y desviación típica: CMJ, RSA y COD.

\begin{tabular}{lccc}
\hline & N & Media & DT \\
\hline CMJ Postest & 6 & 27.35 & 3.17 \\
COD Postest & 6 & 7.67 & .219 \\
RSA Postest & 6 & 8.41 & .287 \\
\hline CMJ: Counter Movement Jump / RSA: Repeated Sprint Ability / COD: Cambios \\
de Dirección V-Cut.
\end{tabular}

$$
c\left(g_{l}\right)=1-\frac{3}{4(n-1)-1}
$$

Para el cálculo de los intervalos de confianza al 95\% (IC95\%), se utilizó el inverso de la varianza $\sigma\left(d_{g 3}\right)$, calculado como:

En el análisis de las diferencias significativas tras la intervención, en la tabla 3 , los resultados muestran que existen diferencias significativas en el caso de la fuerza explosiva, CMJ y en la RSA, $p<.40$ y $p<.008$, respectivamente, es decir que la intervención ha provocado mejora en estas dos variables, mientras que en el caso de los COD no existen diferencias significativas. Si bien hay que señalar que en todos los casos existe mejora en los resultados tras la intervención realizada. Especialmente destacan los resultados obtenidos en la fuerza explosiva con una mejora media de $4.45 \mathrm{~cm}$.

Tabla 3. Diferencias significativas entre pretest y postest.

\begin{tabular}{lcc}
\hline & $\mathrm{t}$ & Sig. \\
\hline $\mathrm{CMJ}$ & -2.760 & $.040^{*}$ \\
\hline COD & 1.740 & .142 \\
\hline $\mathrm{RSA}$ & 4.295 & $.008^{*}$ \\
\hline${ }^{*} p<.05$ & &
\end{tabular}

Por último, la diferencia de medias en el caso del CMJ $(\mathrm{DM}) \pm \mathrm{SD}$ de la toma pretest y postest fue de $-4.45 \pm 3.94$ $\mathrm{cm}$. El TE $[d c 2$ y el IC95\% entre la toma pretest y postest fue de -1.75 SD $(0.19 \&-3.69)$. En el caso de la RSA la DM \pm SD de la toma pretest y postest fue de $0.24 \pm 0.14 \mathrm{sec}$. El TE [dc2] y el IC95\% entre la toma pretest y postest fue de -0.54 SD (-1.09 \& 0). Y, por último, en el caso de la COD La $\mathrm{DM} \pm \mathrm{SD}$ de la toma pre vs. post fue de $0.44 \pm 0.63 \mathrm{sec}$. El TE $[d c 2$ y el IC95\% entre la toma pre y post fue de $-0.54 \mathrm{SD}$ (-1.08 \& 0) (Figura 2). 


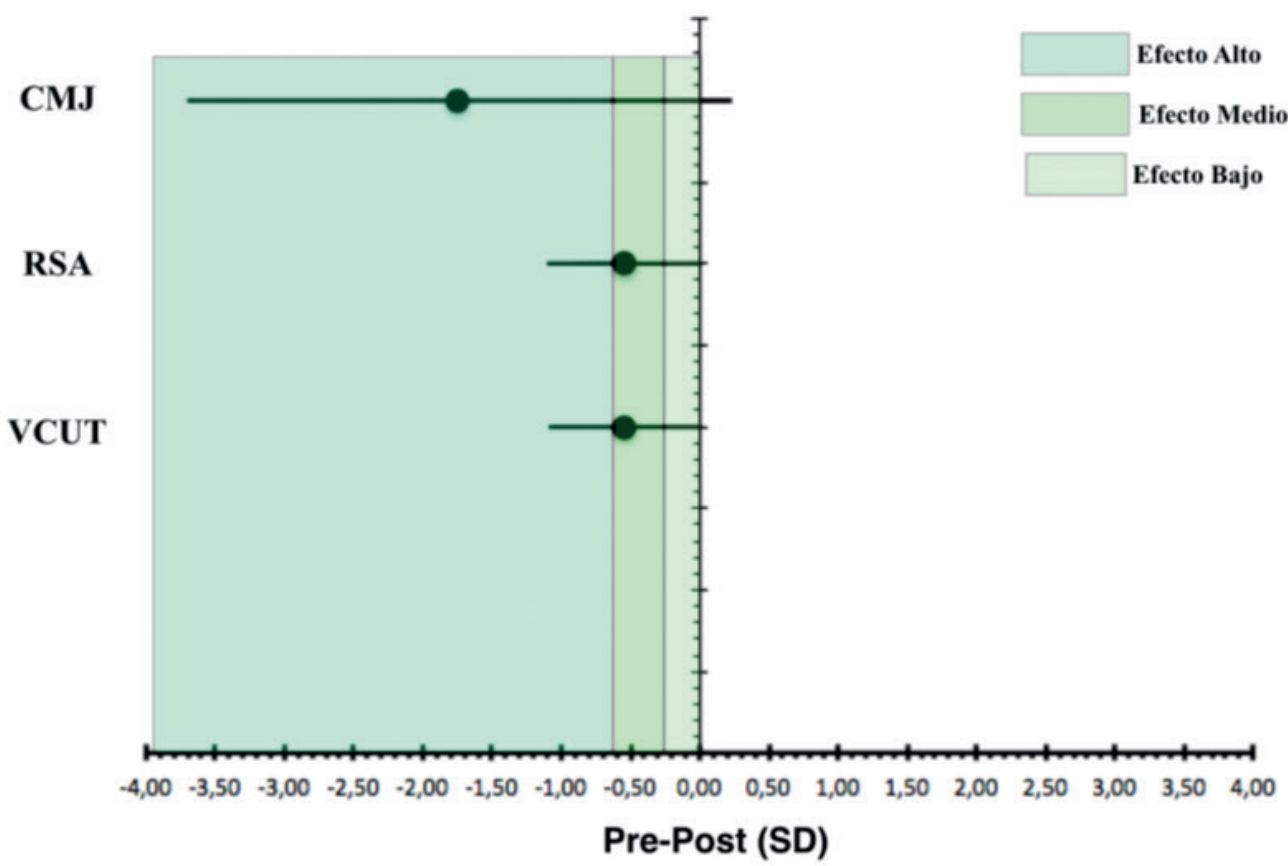

Figura 2. Representación gráfica del tamaño del efecto: CMJ, RSA y VCUT. Se presentan las medias para las diferencias entre los resultados cada uno de los test CMJ ( $\mathrm{p}=.040)$; RSA ( $\mathrm{p}=.008)$; VCUT ( $\mathrm{p}=.14)$.

\section{Discusión}

El objetivo de la investigación fue analizar el efecto de un programa de fuerza mediante tecnología isoinercial durante cuatro semanas en la mejora del rendimiento en mujeres de deportes colectivos con carácter intermitente. Este objetivo ha quedado demostrado ya que se evidencia una mejora en todos los test evaluados, coincidiendo con la investigación de Romero y Tous (2010) en la cual se destaca que hay mejoras después de pocas sesiones de entrenamiento y otras que demuestran la mejora tras la intervención con tecnología isoinercial (Cabanillas et al., 2020; Douglas et al., 2017; Fiorilli et al., 2020; Monajati et al., 2018; Núñez et al., 2016; Petré et al., 2018; Romero et al., 2014).

El efecto del programa de entrenamiento a través de tecnología isoinercial determinó que, se mejoran todas las variables analizadas tanto en los niveles de fuerza, del mismo modo que otros estudios (Cabanillas et al., 2020; De Hoyo et al., 2015; Douglas et al., 2017; Fiorilli et al., 2020; GonzaloSkok, 2017; Núñez et al., 2016; Petré et al., 2018; Romero et al., 2014; Vázquez-Guerrero \& Moras, 2015), como de repetición de esprines, al igual que en el estudio de Romero, et al. (2014) y de los cambios de dirección, del mismo modo que en otros estudios (Cabanillas et al., 2020; Douglas et al., 2017; Fiorilli et al., 2020; Tous-Fajardo et al, 2016) tras la intervención realizada, por lo tanto el entrenamiento isoinercial conduce a mayores mejoras en el rendimiento que el entrenamiento de fútbol tradicional.
Destaca que existen diferencias significativas CMJ (Cabanillas et al., 2020; De Hoyo et al., 2015; Gonzalo-Skok, 2017) y RSA, en la línea con otros estudios (De Hoyo et al., 2015; Monajati et al., 2018; Romero et al., 2014). El RSA está recibiendo una considerable atención por parte de investigadores y profesionales, y en esta línea, los resultados de este programa de entrenamiento a través de tecnología isoinercial determinaron mejoras significativas en los tests RSA, coincidiendo con otros estudios (De Hoyo et al., 2015; Sánchez-Sánchez, Hernández, Marcos, González, Rodríguez \& Carretero, 2014; Monajati et al., 2018). A pesar de que los estudios se han realizado con deportistas amateurs (De Hoyo et al., 2015; Sánchez-Sánchez et al., 2014; Monajati et al., 2018), el primer estudio se centra en jugadores jóvenes, pero de alto nivel eso si con 10 sesiones, el segundo estudio anteriormente citado empleó tan solo dos sesiones de entrenamiento y seis semanas, el tercero, por lo que consideramos que las cuatro semanas de entrenamiento realizadas en este estudio, también son suficientes para mejorar los valores RSA.

Existen diferencias significativas en los tests CMJ y RSA entre hombres y mujeres, pero independientemente del peso, género y especialidad deportiva, aquellos sujetos con valores más elevados en el CMJ tienden a producir mayor potencia en el RSA, como es el caso de nuestra investigación que se relaciona con otro estudio (Balsalobre-Fernández, NevadoGarrosa, Campo-Vecino \& Ganancias-Gómez, 2015; Monajati et al., 2018) que, si bien compara por sexos y alto rendi- 
miento, en el primer caso, no es así en el segundo, ahora bien ambos muestran resultados similares.

El COD es un factor de rendimiento para cualquier deportista de deportes colectivos, aunque parece ser que los factores predictores de rendimiento no están claros. El COD puede definirse como una cualidad física muy compleja que directamente depende de numerosas habilidades motoras como la técnica apropiada, velocidad de sprint recto, fuerza reactiva, fuerza y potencia concéntrica, capacidad para desacelerar y acelerar rápidamente y desequilibrio muscular izquierdo - derecho (Loturco et al., 2017), y que está relacionado con la fuerza excéntrica, la cual ha demostrado ser una cualidad de fuerza importante para un buen rendimiento en el COD (Jones, Bampouras \& Marrin, 2009; Nuñez et al., 2016; Romero et al., 2014; Tous-Fajardo et al., 2016), por lo que, y como ha demostrado este estudio, con un programa de fuerza a través de tecnología isoinercial se pueden obtener mejoras en el test V-Cut / COD, debido al aumento de la potencia muscular en el tren inferior a causa del entrenamiento como en otros estudios que analizan estos efectos, aunque las muestras no son similares (Pérez et al., 2019; Santiago et al., 2015; Yanci et al., 2016), si bien las diferencias no son significativas en nuestro estudio.

En este sentido, sería recomendable que los resultados fueran confirmados en futuras investigaciones tomando un tamaño de muestra mayor, así como comparar con otro grupo de estudio control que no realice el mismo tipo de intervención, como por ejemplo entrenamientos específicos del deporte, entrenamientos de fuerza pliométrica o con uso de chalecos lastrados y aumentar del número de sesiones al doble para comprobar si el efecto es mayor y significativo en todas las variables objeto de estudio, con mayor tiempo de intervención, con más estudios para aumentar el conocimiento en este ámbito de actuación, así como la comparación con otras modalidades deportivas e incluso realizando diferencias de género.

\section{Conclusiones}

Los resultados obtenidos sugieren que el programa de entrenamiento propuesto es un buen método para la mejora tanto en el salto como en la capacidad de repetir sprints de forma significativa, por lo tanto, para la mejora del rendimiento deportivo.

Así pues, y tal y como muestran los resultados obtenidos en este estudio, un programa de entrenamiento de fuerza a través de tecnología isoinercial durante cuatro semanas de entrenamiento es suficiente para la mejora de los cambios de dirección, la resistencia a la repetición de esprines y la fuerza explosiva de piernas.

\section{Referencias}

1. Balsalobre-Fernández, C., Glaister, M., \& Lockey, R. A. (2015). The validity and reliability of an iPhone app for measuring vertical jump performance. Journal of Sports Sciences, 33(15), 1574-1579. http://doi. org/10.1080/02640414.2014.996184.

2. Balsalobre-Fernández, C., Nevado-Garrosa, F., Campo-Vecino, J. \& Ganancias-Gómez, P. (2015). Repetición de esprints y salto vertical en jugadores jóvenes de baloncesto y fútbol de élite. Apunts Educación Fisica y Deportes, 120, 52-57. https://doi.org/10.5672/apunts.2014-0983. es.(2015/2).120.07

3. Bangsbo, J., Mohr, M. \& Krustrup, P. (2006). Physical and metabolic demands of training and match-play in the élite football player. Journal of Sports Science, 24, 665-674.

4. Bishop, D., Spencer, M., Duffield, R., \& Lawrence, S. (2001). The validity of a repeated sprint ability test. Journal of Science and Medicine in Sport, 4(1), 19-29. http://doi.org/10.1016/S1440-2440(01)80004-9

5. Cabanillas, R., Serna, J., Muñoz-Arroyave, V. \& Echeverri, A. (2020). Effect of eccentric overload through isoinertial technology in basketball players. Rev Bras Cineantropom Desempenho Hum, 22, 1-7. DOI: http:// dx.doi.org/10.1590/1980-0037.2020v22e59831

6. Cook, T.D. \& Reichardt, CH. S. (1986). Métodos cualitativos y cuantitativos en investigación evaluativa. Madrid: Morata.

7. De Hoyo, M., Pozzo, M., Sañudo, B., Carrasco, L., Gonzalo-Skok, O., Domínguez-Cobo, S. \& Morán-Camacho E. (2015). Effects of a 10-Week In-Season Eccentric-Overload Training Program on MuscleInjury Prevention and Performance in Junior Elite Soccer Players. Int J Sport Physiol Perform, 10(1), 46-52.

8. Douglas, J., Pearson, S., Ross, A. \& McGuigan, M. (2017). Chronic adaptations to eccentric training: a systematic review. Sports Med, 47(5), 917-941.
9. Fiorilli, G., Mariano, I., Iuliano, E., Giombini, A., Ciccarelli, A., Buonsenso, A., Calcagno, G. \& di Cagno, A. (2020). Isoinertial EccentricOverload Training in Young Soccer Players: Effects on Strength, Sprint, Change of Direction, Agility and Soccer Shooting Precision. Journal of sports science and medicine, 19(1), 213-223.

10. Garganta, J. (2001). Análise da performance nos juegos desportivos. Revisão acerca da análise do juego. Revista Portuguesa de Ciências do Desporto, 1, 57-64.

11. Gonzalo-Skok, O. (2015). La velocidad en el cambio de dirección en los deportes de equipo: Evaluación, especificidad y entrenamiento. Zaragoza. Universidad de Zaragoza. Tesis Doctoral.

12. Gonzalo-Skok, O., Tous-Fajardo, J., Suarez-Arrones, L., Arjol-Serrano, J., Casajús, J., \& Mendez-Villanueva, A. (2015). Validity of the V-cut Test for Young Basketball Players. International Journal of Sports Medicine, 36(11), 893-899. http://doi.org/10.1055/s-0035-1554635

13. Gonzalo-Skok, O., Tous-Fajardo, J., Valero-Campo, C., Berzosa, C., Bataller, A.V., Arjol-Serrano, J.L., et al. (2017). Eccentric-Overload Training in Team-Sport Functional Performance: Constant Bilateral Vertical Versus Variable Unilateral Multidirec-tional Movements. Int J Sport Physiol Perform, 12(7), 951-958. https://dx.doi.org/10.15446/ revfacmed.v64n3.47701

14. Irigoyen, J.Y., Huerta, A. G., Alvira, D. C., Benito, L. Á. R., \& Larumbe, A. L. A. (2014). Evaluación y relación entre distintos parámetros de condición física en futbolistas semi profesionales. Retos: Nuevas Perspectivas de Educación Física, Deporte y Recreación, 26, 114-117.

15. Jones, P., Bampouras, T. M., \& Marrin, K. (2009). An investigation into the physical determinants of change of direction speed. J Sports Med Phys Fitness, 49(1), 97-104.

16. Jones, S. \& Drust, B. (2007). Physiological and technical demands of 
$4 \mathrm{v} 4$ and 8 v 8 games in elite youth soccer players. Kinesiology, 39(2), 150-156.

17. Loturco, I., Pereira, L. A., Moraes, J. E., Kitamura, K., Cal Abad, C. C., Kobal, R., \& Nakamura, F. Y. (2017). Jump-Squat and Half-Squat Exercises: Selective Influences on Speed-Power Performance of Elite Rugby Sevens Players. PLOS ONE, 12(1), 1-11. https://doi.org/10.1371/ journal.pone. 0170627

18. Monajati, A., Larumbe-Zabala, E., Sampson, M.G. \& Naclerio, F. (2018). Injury prevention programs based on flywheel vs. bodyweight resistance in recreational athletes.J Strength Cond Res, 0, 1-9.

19. Norrbrand, L. Pozzo, M. \& Tesch, P.A. (2010). Flywheel resistance training calls for greater eccentric muscle activation than weight training. Eur J Appl Physiol, 110, 997-1005.

20. Núnez, F. J., de Hoyo, M., López, A. M., Sañudo, B., Otero-Esquina, C., Sanchez, H. \& Gonzalo- Skok, O. (2019) Eccentric-concentric Ratio: A Key Factor for Defining Strength Training in Soccer. International Journal of Sports Medicine, 40(12), 796-802.

21. Núnez, V. M., Poblador, C. L. \& Ramírez, J. M. (2016). Entrenamiento muscular a través de tecnología isoinercial en un jugador de fútbol profesional intervenido de rotura total de LCA. Estudio de caso. / Muscle training using isoinertial technology in a professional footballer following surgery for a complete ACL tear. A case study. Retos: Nuevas Perspectivas de Educación Física, Deporte y Recreación, 29, 166-170.

22. Pardeiro, M. \& Yancy, J. (2017). Efectos del calentamiento en el rendimiento físico y en la percepción psicológica en jugadores semi profesionales de fútbol. RYCYDE. Revista Internacional de Ciencias del Deporte, 48(13), 104- 116.

23. Pérez, S., Rodríguez, A., Sánchez, A., De Mena, J. M., Fuentes, J. M., Castańo, R., \& Martín, N. (2019). Efecto de los juegos reducidos sobre jugadoras de fútbol. Revista Internacional de Medicina y Ciencias de la Actividad Física y del Deporte, 19(74), 371-386. https://doi. org/10.15366/rimcafd2019.74.012

24. Pérez, S., Sánchez, J., \& Urchaga, J. D. (2015). The reasons for participation in football: A study by category and level of competition players. Cultura, Ciencia y Deporte, 10(30), 187-198. https://doi.org/10.12800/ ccd.v10i30.588

25. Petré, H., Wernstål, F., \& Mattsson, C. M. (2018). Effects of Flywheel Training on Strength-Related Variables: A Meta-analysis. Sports Medicine, 4(1), 55-70. https://doi.org/10.1186/s40798-018-0169-5

26. Pita, S. \& Pértega, S. (2002). Investigación: Investigación cuantitativa y cualitativa. Cad Aten Primaria, 9, 76-78.

27. Prieto-Mondragón, L.P., Camargo-Rojas, D. A. \& Quiceno, C. A. (2016). Isoinertial technology for rehabilitation and prevention of muscle injuries of soccer players: literature review. Revista de la Facultad de Medicina, 64(3), 543-550.
28. Prieto, Y. H. H., \& García, J. M. (2013). Efectos de un entrenamiento específico de potencia aplicado a futbolistas juveniles para la mejora de la velocidad con cambio de dirección. European Journal of Human Movement, (31), 17-36.

29. Romero, D. \& Tous, J. (2010). Prevención de lesiones en el deporte. Madrid: Editorial Médica Panamericana S.A

30. Romero, S., Feria, A., Sañudo, B., De Hoyo, M., \& Del Ojo, J. J. (2014). Efectos de entrenamiento de fuerza en sistema isoinercial sobre la mejora del CMJ en jóvenes futbolistas de elite. / Effects of strength training using a isoinertial device on jump ability in young elite soccer players. Retos: Nuevas Perspectivas de Educación Física, Deporte y Recreación, 26, 180-182.

31. Sánchez-Sánchez, J., Hernández, C., Marcos, V., González, A., Rodríguez, A. \& Carretero, M. (2016). Efecto de un entrenamiento intermitente con y sin cambios de dirección, sobre el rendimiento físico de jóvenes futbolistas. Retos: Nuevas Perspectivas de Educación Física, Deporte $y$ Recreación, 30, 70-75.

32. Santiago, A., Granados, C., Quintela, K. \& Yanci, J. (2015). Diferen cias entre jugadores de fútbol de distintas edades en la capacidad de aceleración, cambio de dirección y salto. Cultura, Ciencia y Deporte, 10, $135-143$.

33. Suarez-Arrones, L., de Villarreal, E. S., Núńez, F. J., Di Salvo, V., Petri, C., Buccolini, A. \& Mendez-Villanueva, A. (2018) In-season eccentricoverload training in elite soccer players: Effects on body composition, strength and sprint performance. PloS One, 13(10), 1-16.

34. Suarez-Arrones, L., Gonzalo-Skok, O., Carrasquilla, I., Asian-Clemente, J., Santalla, A., Lara-Lopez, P., \& Nunez, F.J. (2020). Relationships between Change of Direction, Sprint,Jump, and Squat Power Performance. Sports, 8(3), 1-10. doi:10.3390/sports8030038.

35. Tous-Fajardo, J., Gonzalo-Skok, O., Arjol-Serrano, J. L., \& Tesch, P. (2016). Enhancing Change-of-Direction Speed in Soccer Players by Functional Inertial Eccentric Overload and Vibration Training. International Journal of Sports Physiology and Performance, 11(1), 66-73. https://doi.org/10.1123/ijspp.2015-0010

36. Vázquez-Guerrero, J. \& Moras, G. (2015). Cambios en la arquitectura muscular y en la velocidad de ejecución de sentadillas en VersaPulley en condiciones estables e inestables en jugadores junior de baloncesto de élite durante una temporada. Cuadernos de Psicología del Deporte, 15(3), 243-252. https://doi.org/10.4321/S157884232015000300029

37. Yanci, J., Castillo, D., Vizcay, J.J., Pitillas, I. \& Iturricastillo, A. (2016). Relación entre la capacidad de aceleración, cambio de dirección y salto horizontal en atletas jóvenes. RICCAFD, Revista Iberoamericana de la Actividad Fisica y el Deporte, 5(2), 1-15. 